\title{
Correlation between Process Parameters and Mechanical Properties in Parts Printed by the Fused Deposition Modeling Process
}

\author{
Samuel Attoye ${ }^{1}$, Ehsan Malekipour ${ }^{2, *}$, Hazim El-Mounayri ${ }^{3}$ \\ Collaborative Additive Manufacturing Research Initiative at IUPUI (CAMRI) \\ Purdue School of Engineering and Technology, Indianapolis, IN, USA \\ soattoye@umail.iu.edu ${ }^{1}$,emalekip@purdue.edu ${ }^{2}$,helmouna@iupui.edu ${ }^{3}$
}

\begin{abstract}
Fused deposition modeling (FDM) represents one of the most common techniques for rapid prototyping and industrial additive manufacturing (AM). Optimizing the process parameters which significantly impact the mechanical properties is critical to achieving the ultimate final part quality sought by industry today. This work investigates the effect of different process parameters including nozzle temperature, printing speed, and print orientation on Young's modulus, yield strength, and ultimate strength of the final part for two types of filament, namely, Poly Lactic Acid (PLA) and Acrylonitrile Butadiene Styrene (ABS). Design of Experiments (DOE) is used to determine optimized values of the process parameters for each type of filaments; also, a comparison is made between the mechanical properties of the parts fabricated with the two materials. The results show that Y-axis orientation presents the best mechanical properties in PLA while $\mathrm{X}$-axis orientation is the best orientation to print parts with ABS.
\end{abstract}

Keywords: Additive manufacturing; fused deposition modeling; process parameters; mechanical properties

\section{Introduction}

Fused deposition modeling (FDM) is a subset technology of additive manufacturing by material extrusion. FDM is devised and patented by Scott Crump [1] and Industrial Technology Research Institute_(ITRI) ${ }^{\dagger}$ stated that this emerging technology, represents one of the most common techniques for prototyping and personal additive manufacturing (AM). FDM also produces end-use parts fit for mechanical, chemical and biochemical operating conditions. This technology, however, is not free from limitation. In this process there is an instant hardening of the plastic as it bonds to the layer below and another layer is deposited by the extrusion nozzle. In this procedure, the properties of the deposited material, the printer specifications especially in 3D printing finely-detailed items, and the range of process parameters affect significantly on the finished product quality. Any depletion in these categories leads to some potential defects such as unfavorable mechanical properties, poor surface condition, etc. Thus, scholars have focused in these fields to improve the ultimate quality of the fabricated parts. In an effort, Dorigato et al (2017) included reinforcing particles (i.e., Nano-fillers) with the polymer to improve the mechanical properties of FDM final products. It was observed that this approach significantly boost the properties such as impact resistance, elastic modulus, thermal stability, and fire resistance, compared with those of neat polymers [2].

In a different effort, Keng Hsu at the Arizona State University improved the interfacial strength by preheating the base layer [3]. On the other hand, optimization of the process parameters is considered an important alternative for improving the quality of the final parts. Several efforts are underway to improve the mechanical properties of FDM parts; for an instance, in a study by Onwubolu and Rayegani, a functional relationship between process parameters and tensile strength for FDM process was developed using group method for data modeling. In this study, the effect of layer thickness, part orientation, raster angle, raster width, and air gap on the tensile strength of test specimen was studied [4]. In another study, Stephen A., et al (2014) identified the tests that can be most sensitive to the changes in processing conditions and

\footnotetext{
${ }^{*}$ Corresponding author

${ }^{+}$https://www.itri.org.tw
} 
differences in interlayer bond strength which affect the quality of fabricated parts. The various mechanical properties of tensile, flexural properties, notched and un-notched impact strengths and the influence of changes in processing parameters and orientations of specimens on the quality was investigated. Analysis of variance (ANOVA) was used to compare the significance of the effect of processing parameters on the mechanical properties. Their conclusion, on the basis of the data from experiments, was that a combination of measures of modulus, weight and impact strength can be used to infer the quality of a build on an FDM machine [5].

Previous works investigated the effects of different materials or changing process parameters on mechanical properties; however, there are still gaps in literature relating to the comparison between the obtained mechanical properties for the part printed by prevalent materials in FDM process and also, introducing the optimized values for process parameters which lead to the best mechanical properties for the parts printed by each material. In this work, we investigate the influence of printing orientation, nozzle temperature, and printing speed on Young's modulus, yield strength, and ultimate strength of a modified ASTM tensile strength test standard part (ASTM D638) [6] for two types of material, namely, Poly Lactic Acid (PLA) and Acrylonitrile Butadiene Styrene (ABS), used predominantly in FDM process. Printing orientation plays a significant role in the ultimate mechanical properties since it affects the bonding between layers. As a matter of fact, the anisotropy of the fabricated parts has been always a fundamental problem in FDM and thus, selecting the right printing orientation according to the function of the part is crucial. The nozzle temperature also affects the quality of the part directly. This temperature was adjusted based on the recommended temperature on each type of filament rolls. Effects of changing in printing speed are also investigated and depicted in our work. As it already mentioned, we conducted our experiments for both PLA and ABS.

\section{Methodology and experimental setup}

The interaction between printing parameters (nozzle temperature, printing speed, and print orientation) adjusted during fabrication and the mechanical properties (Young's modulus, yield strength, and ultimate tensile strength) was observed. The specimen selected and modified (Fig. 1) for monitoring and evaluation is based on the ASTM tensile strength test standard part (ASTM D638). The dimensions followed by minor adjustments to facilitate the printing process.

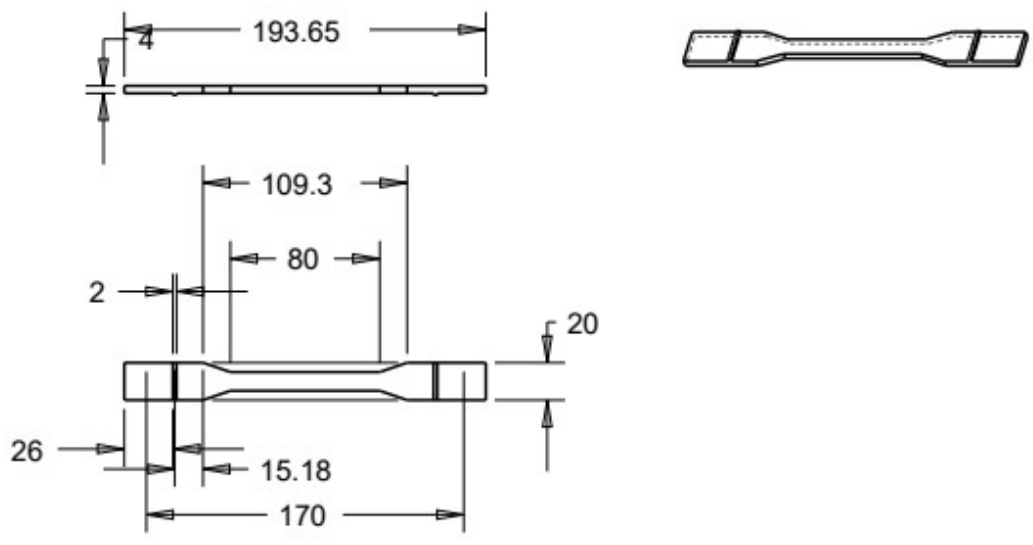

Figure 1: The modified ASTM tensile strength sample used for experiments

Two sets of the specimen were fabricated. Set-A consisted of thirty-six (36) individual printed MakerBot PLA specimens (specimen A1 to A36). Set-B consisted of twenty-seven (27) individual printed Filabot ABS specimens. The printing process parameters (nozzle temperature, printing speed), adjusted for 
each part, are listed in Table 1. The printing parameter values were selected based on the machine and material specifications. Three nozzle temperature values (low, medium and high) were considered for each filament according to the recommended temperature of the filament rolls. Various build orientations used in the experiments are shown in Fig.2. The X, Y, Z orientations were used in the fabrication of both set-A and set-B specimens; while the 45-degree orientation was only used for set-A specimens (Fig. 2).

Table 1: printing parameters adjusted for fabrication process of set-A specimen A1-A36 and set-B specimen B1-B27

\begin{tabular}{|c|c|c|c|c|c|c|}
\hline Printing parameters & \multicolumn{3}{|c|}{ Values for printer A } & \multicolumn{3}{c|}{ Values for printer B } \\
\hline Nozzle temperature in ${ }^{\circ} \mathrm{C}$ & 200 & 215 & 220 & 215 & 225 & 235 \\
\hline Printing speed in $\mathrm{mm} / \mathrm{s}$ & 20 & 40 & 60 & 20 & 40 & 60 \\
\hline
\end{tabular}

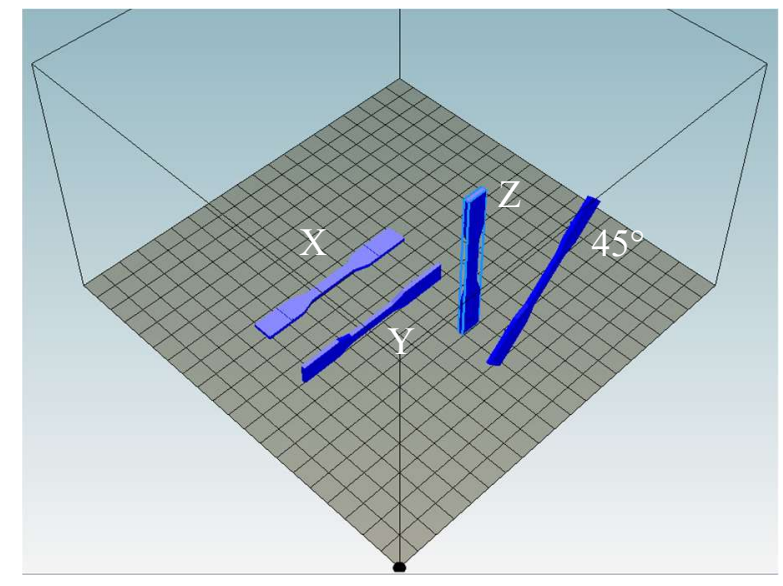

Figure 2: $\mathrm{X}, \mathrm{Y}, \mathrm{Z}$, and $45^{\circ}$ orientation used in fabrication of specimen set-A and $\mathrm{X}, \mathrm{Y}$, and $\mathrm{Z}$ orientation used in fabrication of set-B

After the specimens were printed, the tensile strength test was performed on each part. The results were analyzed and revealed in section 3. Mechanical analysis of set-A and set-B was performed using Q-test machine, Matt Struve Demo MTS Extensometer, and test-works software. Young's modulus, yield strength, and ultimate strength of the FDM printed specimens were obtained.
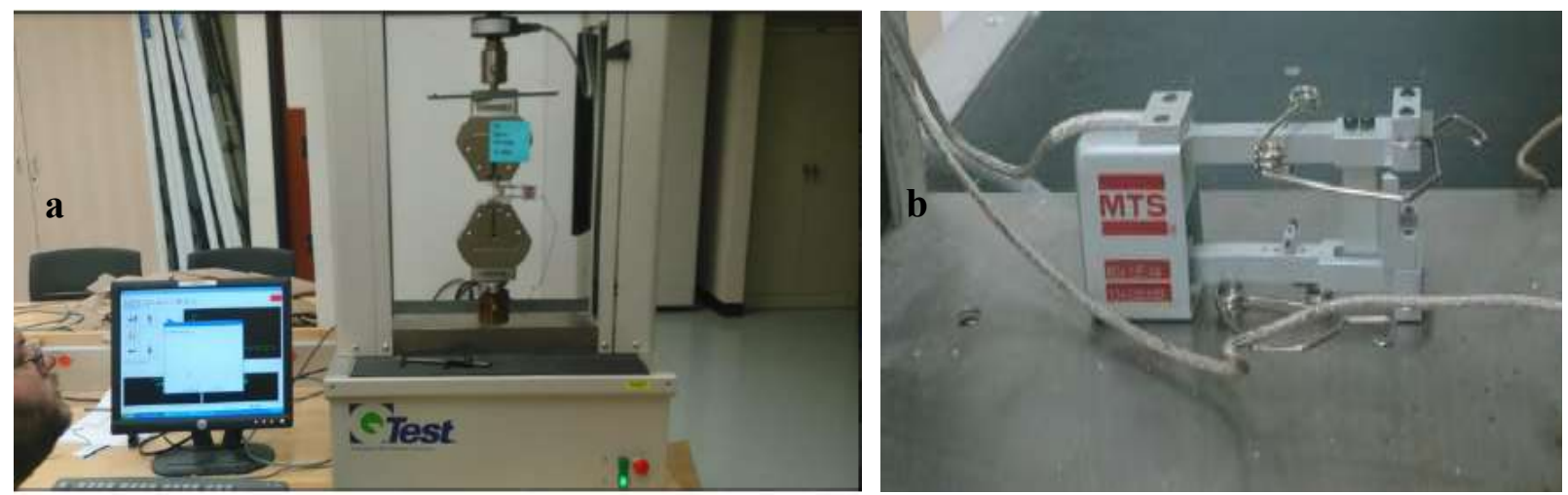

Figure 3: (a) Q-Test machine (b) Matt Struve Demo MTS Extensometer 


\section{Results and discussion}

During the printing process, it was observed that the specimens fabricated in the $\mathrm{X}$ orientation took the shortest time to print for both set-A and set-B specimens. This was due to the fewer layers required to complete the part built in this orientation. For set-A specimens, printing at the 45-degree orientation took the longest time due to the requirement of support structures. On the other hand, printing at the Z-axis orientation took the longest time for set-B specimens. This was due to the requirement of support structures and the specimen height. The effect of the printing speed on the build time was not significant; however, the mechanical analysis shows a more discernable influence of the printing speed parameter on the build process and final product mechanical properties.

Almost all the specimens printed with either PLA or ABS (set-A or set-B) in the X and Y orientations tended to fracture at location close to the end of the sample, at the "neck" region; however, around 33\% of the specimens in both sets printed in the z-orientation, tended to fracture at location that is closer to the mid-region of the specimens and $66 \%$ close to the end of the samples. In the specimens printed at 45 -degree in set-A, the fracture spot tends to shift to the middle for all the process parameters. Some specimens after evaluation on the Q-test machine are depicted in Fig. 4.
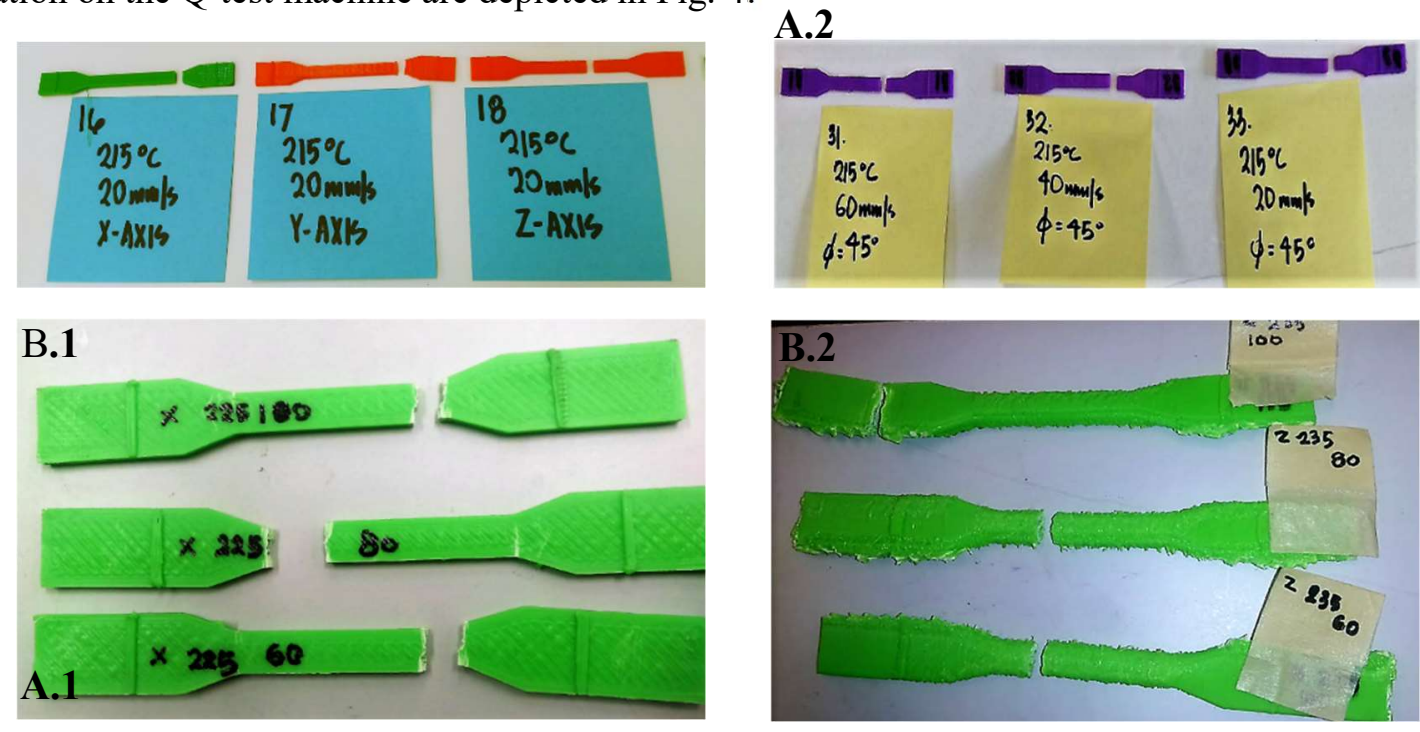

Figure 4: A.1 specimens printed in X, Y, Z orientation with PLA; A.2 specimens printed in $45^{\circ}$ with PLA B. 1 specimens printed in $X$ orientation with ABS; B.2 specimens printed in $Z$ orientation with $A B S$

The effects of changing the printing parameters, i.e. nozzle temperature, printing speed, and printing orientations, on the mechanical behavior of each specimen are investigated. In this regard, values of Young's modulus, yield strength, and ultimate tensile strength of the printed specimens in X, Y, Z, and 45degree directions for PLA filament and X, Y, and Z for ABS filament for different nozzle temperatures and printing speeds gotten from the tensile strength and are presented in the sections 3.1 and 3.2.

\subsection{Mechanical properties of the specimens fabricated with PLA filament in $\mathrm{X}, \mathrm{Y}, \mathrm{Z}$, and $45^{\circ}$}

Figs 5-7 show respectively Young's modulus, yield strength, and ultimate tensile strength of the fabricated PLA (set-A) specimens. It can be observed from Figs. $5-7$ that, the specimens printed in the Y-axis orientation show the highest mechanical properties compared with the specimens printed in the $\mathrm{X}, \mathrm{Z}$-axis and 45-degree orientation. In these samples, the minimum value of mechanical properties in Y-direction is predominantly higher than the maximum one in the other directions. Therefore, $\mathrm{Y}$ is the best direction suggested for printing with PLA. For all the mechanical properties, 45-degree direction shows the weakest properties; thus, this direction is not recommended for printing of parts. The diagrams also depict that the 
highest values of almost all the mechanical properties were obtained at the lowest temperature which is 200 ${ }^{\circ} \mathrm{C}$ and the highest printing speed which is $60 \mathrm{~mm} / \mathrm{s}$ for all $\mathrm{X}, \mathrm{Y}$, and $\mathrm{Z}$ orientations. Increasing the temperature weakens the mechanical properties for this speed. Changing the nozzle temperature does not show any specific trend on changing of mechanical properties for the other printing speeds.

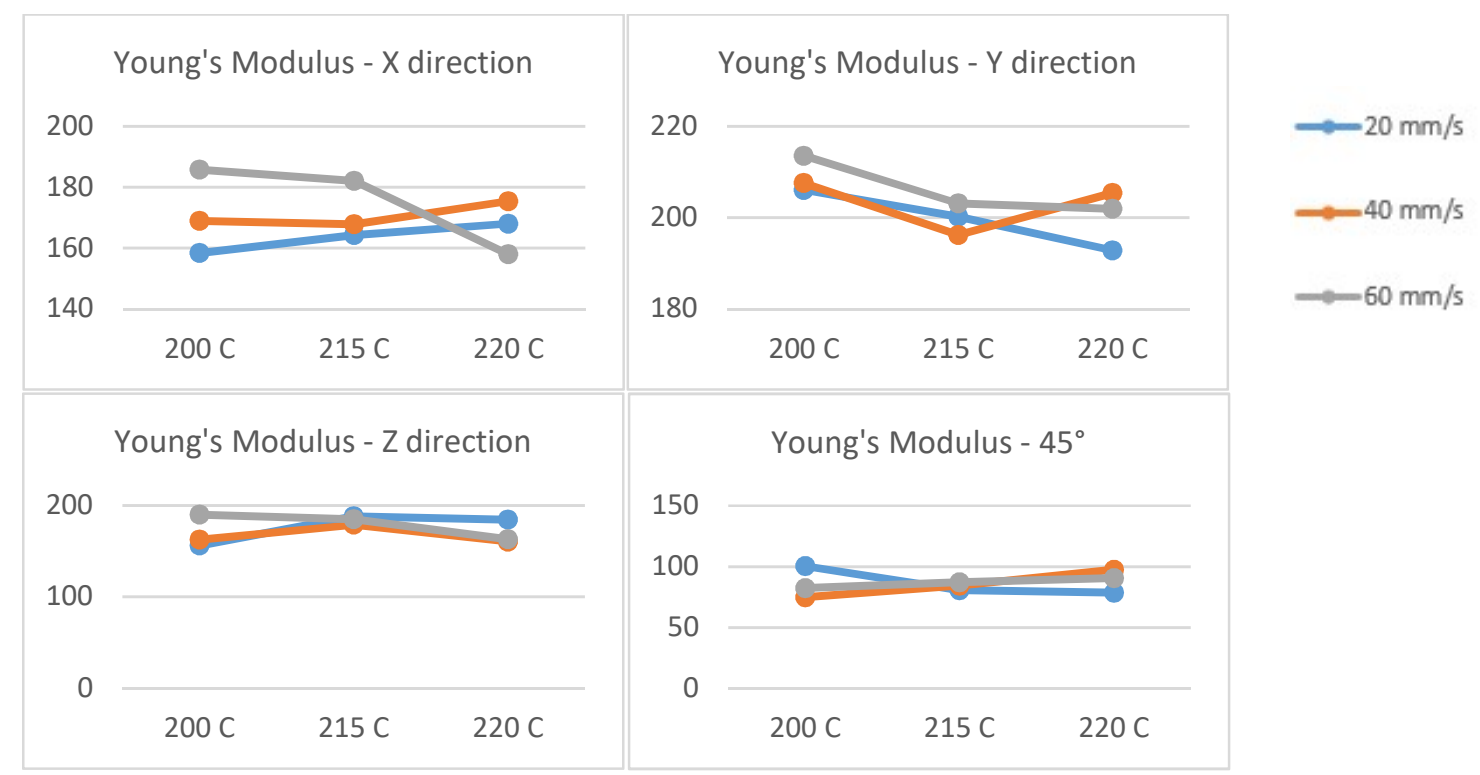

Figure 5: Young's modulus in X, Y, Z orientations and $45^{\circ}$ for the part printed with PLA in different nozzle temperatures and printing speeds

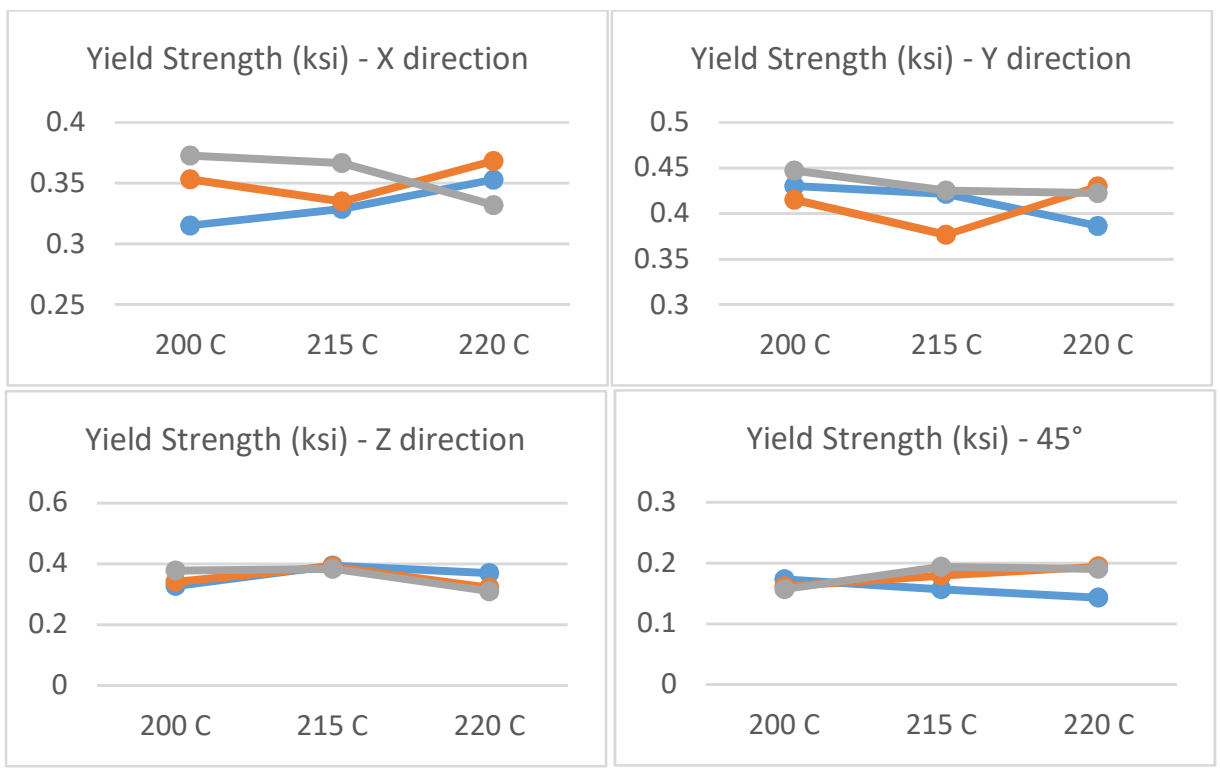

Figure 6: Yield strength in $\mathrm{X}, \mathrm{Y}, \mathrm{Z}$ orientations and $45^{\circ}$ for the part printed with PLA in different nozzle temperatures and printing speeds 


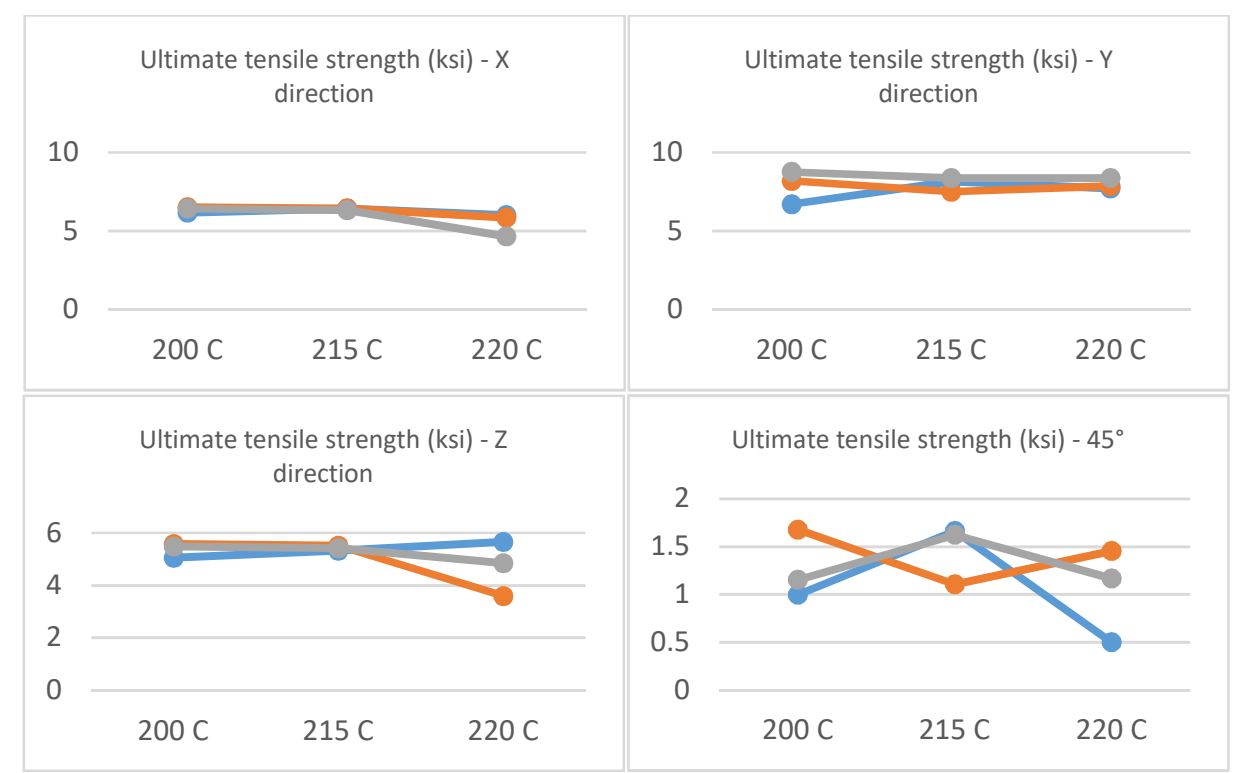

Figure 7: Ultimate tensile strength in $\mathrm{X}, \mathrm{Y}, \mathrm{Z}$ orientations and $45^{\circ}$ for the part printed with PLA in different nozzle temperatures and printing speeds

\subsection{Mechanical properties of the specimens fabricated with $A B S$ filament in $X, Y, Z$}

For ABS filament, the effect of altering nozzle temperature and printing speed on the mechanical properties of fabricated parts in the three main directions i.e. $\mathrm{X}, \mathrm{Y}$, and $\mathrm{Z}$ was investigated. We did not investigate the mechanical properties in 45 degree for ABS material, as this orientation showed the weakest properties for PLA. The obtained Young's modulus, yield strength, and ultimate tensile strength of the fabricated ABS (set-B) specimens are shown in Figs. 8-10. The results indicate that the part printed in $\mathrm{X}$ and $\mathrm{Z}$ orientations have better mechanical properties compared with the parts printed in $Y$ orientation. In these samples, the highest Young's modulus achieved for the parts printed in $X$ orientation; however, the maximum yield strength and ultimate strength achieved for the parts printed in either X or Z (however Z shows a bit better properties). By considering of Young's modulus, $\mathrm{X}$ is the best orientation suggested for printing of a part with ABS. The diagrams also depict that the highest mechanical properties in X-direction were obtained at the temperature of $225^{\circ} \mathrm{C}$ and the printing speed of $20 \mathrm{~mm} / \mathrm{s}$. Increasing or decreasing of the temperature will weaken the mechanical properties significantly. While the best mechanical properties for Z-direction were predominantly obtained at the temperature of $235^{\circ} \mathrm{C}$ and the printing speed of $40 \mathrm{~mm} / \mathrm{s}$. However, the Yield strength shows a better result for the temperature at $215^{\circ} \mathrm{C}$ and the printing speed of $20 \mathrm{~mm} / \mathrm{s}$. It is noteworthy that the weakest mechanical properties obtained for $\mathrm{Z}$ direction and for the temperature at $235^{\circ} \mathrm{C}$ and the printing speed of $20 \mathrm{~mm} / \mathrm{s}$.

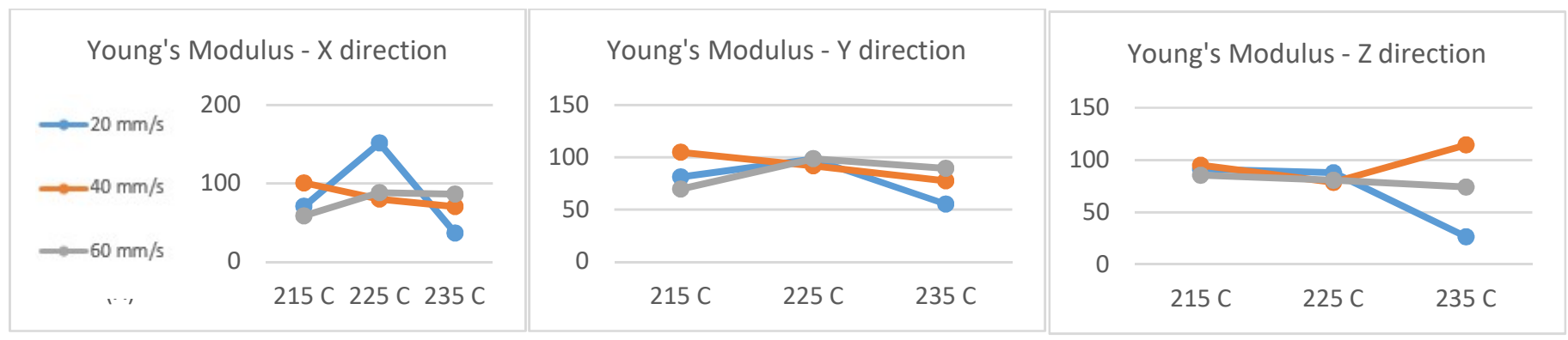

Figure 8: Young's modulus in $\mathrm{X}, \mathrm{Y}$, and $\mathrm{Z}$ orientations for the part printed with $\mathrm{ABS}$ in different nozzle temperatures and printing speeds 


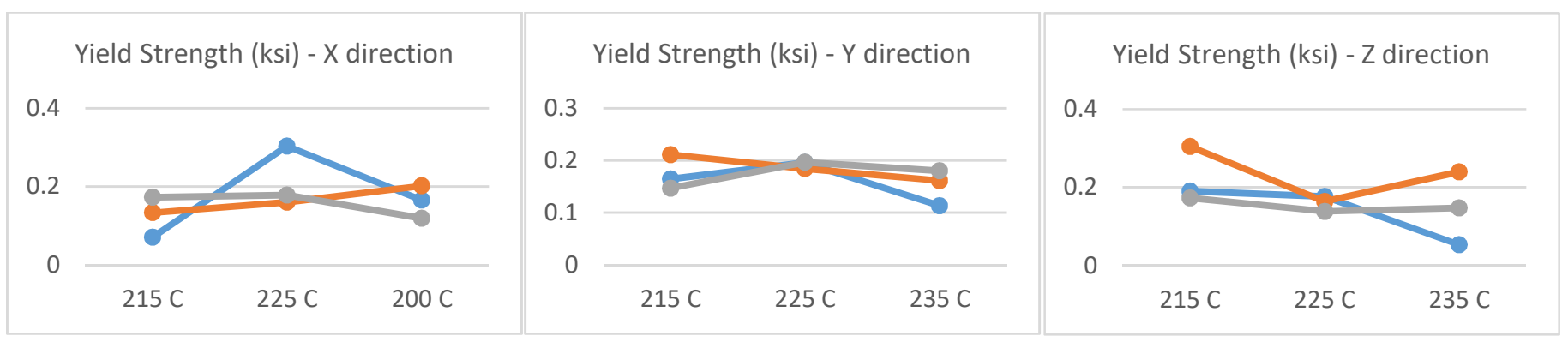

Figure 9: Yield strength in $\mathrm{X}, \mathrm{Y}$, and $\mathrm{Z}$ orientations for the part printed with $\mathrm{ABS}$ in different nozzle temperatures and printing speeds

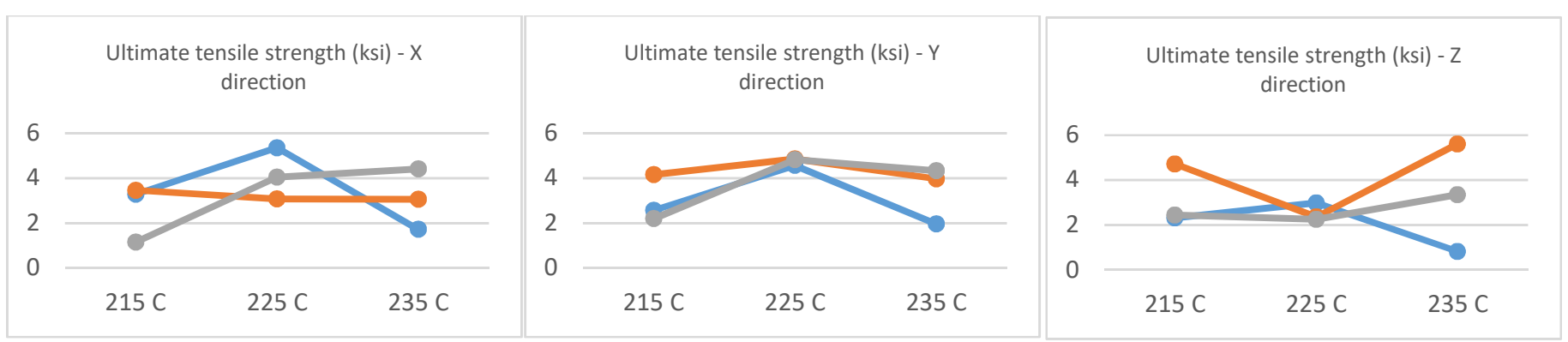

Figure 10: Ultimate tensile strength in $\mathrm{X}, \mathrm{Y}$, and $\mathrm{Z}$ orientations for the part printed with $\mathrm{ABS}$ in different nozzle temperatures and printing speeds

Fig. 11 compares the maximum mechanical properties obtained for the specimens printed with PLA and ABS in different orientations. As this figure depicted, PLA shows stronger mechanical properties compared with ABS.

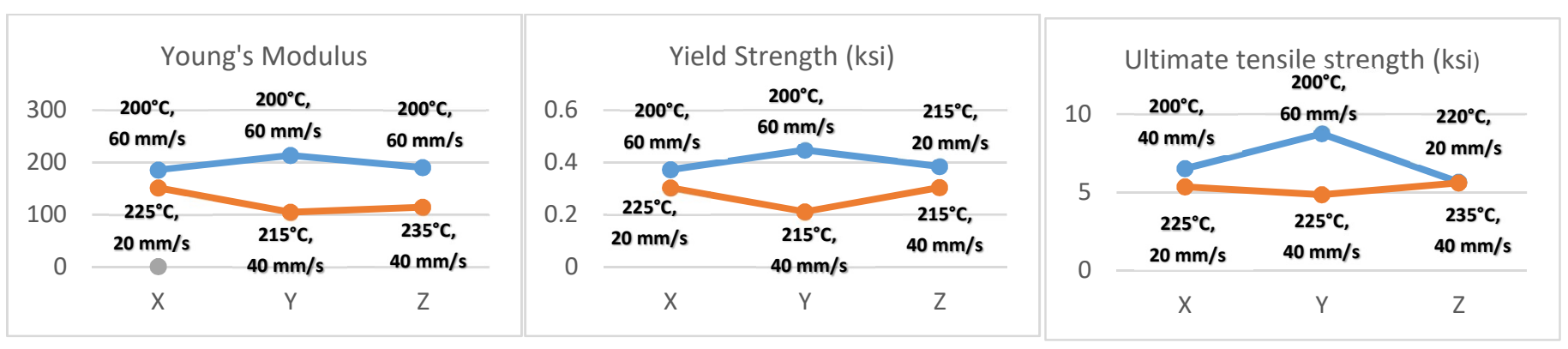

Figure 11: comparison between the maximum mechanical properties of the part printed with PLA and ABS

\section{Conclusions and future works}

In this research, the effect of printing parameters on the mechanical properties of the PLA and ABS specimens produced by fused deposition modeling process was investigated. The work reveals that PLA shows stronger mechanical properties compared with ABS. These experimental results also suggest that the specimens printed in the $\mathrm{Y}$-axis orientation present the best mechanical properties in PLA while X-axis orientation is the best orientation to print parts with ABS. The optimized process parameters are recommended for the tested materials in the revealed range of parameters. More specimens need to be printed for each set of parameters to measure the experimental error. Furthermore, the obtained experimental data needs to be analyzed and explained scientifically. 


\section{References}

[1] E. Matias and B. Rao, "3D printing: On its historical evolution and the implications for business," in Management of Engineering and Technology (PICMET), 2015 Portland International Conference on, 2015, pp. 551-558.

[2] A. Dorigato, V. Moretti, S. Dul, S. Unterberger, and A. Pegoretti, "Electrically conductive nanocomposites for fused deposition modelling," Synthetic Metals, vol. 226, pp. 7-14, 2017.

[3] A. K. Ravi, A. Deshpande, and K. H. Hsu, "An in-process laser localized pre-deposition heating approach to inter-layer bond strengthening in extrusion based polymer additive manufacturing," Journal of Manufacturing Processes, vol. 24, pp. 179-185, 2016.

[4] G. C. Onwubolu and F. Rayegani, "Characterization and optimization of mechanical properties of ABS parts manufactured by the fused deposition modelling process," International Journal of Manufacturing Engineering, vol. 2014, 2014.

[5] A. Stephen, K. Dalgarno, and J. Munguia, "Quality assurance and process monitoring of fused deposition modelling made parts," in High Value Manufacturing: Advanced Research in Virtual and Rapid Prototyping: Proceedings of the 6th International Conference on Advanced Research in Virtual and Rapid Prototyping, 2013, pp. 31-35.

[6] S.-H. Ahn, M. Montero, D. Odell, S. Roundy, and P. K. Wright, "Anisotropic material properties of fused deposition modeling ABS," Rapid prototyping journal, vol. 8, pp. 248-257, 2002. 